\title{
Betriebliche Integration von Geflohenen
}

\section{Ergebnisse einer explorativen qualitativen Studie}

\author{
Irma Rybnikova@ und Stefanie Wilkmann
}

Hochschule Hamm-Lippstadt

\begin{abstract}
Zusammenfassung: Während die vorliegende Forschung zur betrieblichen Integration von Geflohenen in Deutschland mehrheitlich auf die Unternehmensperspektive ausgerichtet ist, kommt die Sicht der Geflohenen oftmals zu kurz. In dieser explorativen Studie auf Basis von qualitativen Interviews mit erwerbstätigen Geflohenen untersuchen wir die fachliche, soziale und werteorientierte betriebliche Integration. Dabei stellen wir ein großes Defizit von unternehmensinitiierten Unterstützungsangeboten fest, wie der betriebsspezifische Sprachunterricht. Wir thematisieren dabei auch die gravierende Bedeutung von informeller Integrationsarbeit seitens der Arbeitskolleginnen und -kollegen und formulieren Gestaltungsempfehlungen für Betriebe.
\end{abstract}

Schlüsselwörter: Geflohene, betriebliche Integration, qualitative Forschung

Work-Based Integration of Refugees: Findings From an Exploratory Qualitative Study

Abstract: While most of the research regarding workplace integration of refugees in Germany has focused on the employers' point of view, the perspective of the refugees has often remained neglected. In this exploratory study, based on qualitative interviews with employed refugees, we investigate professional, social, and value-oriented workplace integration into companies. We identify a major deficit in company-initiated support measures such as company-specific language training. Finally, we address the relevance of informal work integration as carried out by rank-and-file employees, and formulate recommendations for companies.

Keywords: refugees, workplace integration, qualitative research

Zusammen mit den rapide gestiegenen Asylanträgen in Deutschland in den Jahren 2015 und 2016 erlangte auch die arbeitsmarktbezogene Integration von Geflohenen mediale, politische und wirtschaftliche Relevanz. Die Beschäftigungszahl der hauptsächlich aus den Ländern Pakistan, Eritrea, Nigeria, Iran, Islamische Republik, Afghanistan, Somalia, Irak und Syrien geflohenen Personen lag in Deutschland im Oktober 2014 bei 71 000, zwei Jahre später zählte man 125000 beschäftigte Geflohene und im Januar 2020 wurden 360000 Personen sozialversicherungspflichtig beschäftigt, zudem gingen 74000 weitere einer geringfügigen Beschäftigung nach (vgl. Bundesagentur für Arbeit, 2020, S. 14). Dabei sind die schulischen und die beruflichen Qualifikationen von Geflohenen, die sie aus ihren Heimatländern vorweisen, äußerst heterogen: Neben den Personen mit Hochschulabschlüssen und Promotionen trifft man auch auf jene ohne nachweisbare Ausbildung oder Schulabschlüsse (Brücker et al., 2018). Tendenziell wird unter den Geflohenen aber eine geringere bzw. seltenere Hochschulbildung und Be- rufsbildung als der deutsche Bevölkerungsdurchschnitt festgestellt (Brücker et al., 2019, S. 8).

Inzwischen liegt auch eine Reihe von Forschungsstudien zur arbeitsmarktbezogenen Integration von Geflohenen in Deutschland vor. Oftmals handelt es sich dabei um Betrachtungen von Herausforderungen aus der Unternehmensperspektive, sowohl im Vorfeld eines beruflichen Einstiegs als auch während einer Beschäftigung von Geflohenen. Allen voran werden sprachliche Barrieren angesprochen (z.B. Büschel et al., 2018; Meier \& Mager, 2019; Gürtzgen et al., 2017), kulturelle Besonderheiten, wie eine abweichende Arbeitsmentalität (z.B. Blunck, 2018; Flake et al., 2017b), oder die Schwierigkeit, fachliche Kompetenzen von Geflohenen einzuschätzen aufgrund der fehlenden Nachweise über Ausbildungsabschlüsse (z. B. Büschel et al. 2018; Gürtzgen et al., 2017). In den letzten Jahren wurden zudem quantitative Erhebungen durchgeführt, in denen die Arbeitsmarktintegration von Geflohenen untersucht wurde (Brücker et al., 2018; Brücker et al., 2019). Hier standen solche Aspekte 
im Fokus, wie individuelle Qualifikationen, Sprachniveau, Ausbildung in Deutschland.

Was in der bisherigen Forschung jedoch zu kurz kommt, ist zum einen die Untersuchung der Geflohenensicht auf das Integrationsgeschehen und zum anderen eine dezidierte Analyse von betrieblichen Integrationspraktiken im Fall von Geflohenen. In der vorliegenden Studie wollen wir daher mit Hilfe von qualitativen Interviews mit Geflohenen eruieren, inwiefern Maßnahmen in Unternehmen ergriffen werden, um die Integration der Geflohenen am Arbeitsplatz zu ermöglichen, und wie diese Integrationsangebote durch die Geflohenen wahrgenommen und bewertet werden.

\section{Forschungsstand}

Die vorliegende Literatur in Bezug auf die betriebliche Integration von Geflohenen befasst sich vorwiegend mit der Perspektive der Unternehmen. Im Vordergrund steht dabei die Frage, inwiefern Betriebe der Beschäftigung von Geflohenen gegenüber aufgeschlossen sind. Studien, die hauptsächlich auf quantitativen Befragungen von Unternehmen beruhen, verdeutlichen, dass die Betriebe den Geflohenen gegenüber zwar durchaus aufgeschlossen sind, gleichzeitig aber auch von Herausforderungen in Verbindung mit sprachlichen Schwierigkeiten und kulturellen Differenzen berichten (OECD, 2017; Eilers et al., 2017; Gürtzgen et al., 2017; Büschel et al., 2018). Diese Probleme gelten auch als wesentliche Gründe für eine bisher recht niedrig gebliebene Beschäftigungserfahrung mit Geflohenen in deutschen Betrieben, die je nach Studie zwischen 10 Prozent (Gürtzgen et al., 2017) und 25 Prozent (Flake et al., 2017b) aller betrachteten Unternehmen variiert, davon insbesondere in der Arbeitnehmerüberlassungsbranche. Zur Verbesserung der Situation erwarten Unternehmen umfangreiche öffentlich geförderte Maßnahmen, die insbesondere eine sprachliche Förderung für Geflohene, aber auch eine fachliche Weiterbildung für diese Zielgruppe vorsehen (Eilers et al., 2017; OECD, 2017, S. 57). Was Unternehmen selbst an Maßnahmen zur Arbeitsmarktintegration von Geflohenen ergreifen, haben Müller und Schmidt (2016) in ihrer fallstudienbasierten Arbeit betrachtet. Sie stellen dabei fest, dass Unternehmen einerseits ihre Beschäftigten für ehrenamtliche Engagements hinsichtlich der Integration von Geflohenen freistellen sowie für gesellschaftliche Initiativen spenden. Andererseits erfolgt die betriebliche Integration von $\mathrm{Ge}$ flohenen in Form von Sprachkursen, Praktika oder berufsvorbereitenden Maßnahmen (vgl. Müller \& Schmidt, 2016, S. 7).
Während in den genannten Studien die Perspektive der Unternehmensvertreterinnen und Unternehmensvertreter auf die betriebliche Integration von Geflohenen dominiert, findet man darüber, wie die Geflohenen selbst die betrieblichen Integrationsmaßnahmen erleben, deutlich weniger Material. Kritisch wird in der Forschung im Fall von Geflohenen praktizierte Beschäftigungsmodelle thematisiert, wie Praktika oder befristete Arbeitsverträge (vgl. Flake et al., 2017a, S. 8), und daraus resultierende oft niedrige Entlohnung (vgl. Battisti et al., 2015; Lundborg \& Skedinger, 2016; Brücker et al., 2019). Brücker et al. (2016, 2018) untersuchen im Rahmen der IAB-BAMFSOEP-Befragung (erste Welle) verschiedene individuelle Aspekte, wie Fluchtmotive, Erwerbsbiographien, Sprachkompetenzen, Erwerbsstatus sowie Werte (vgl. Brücker et al., 2016) und kognitive Fähigkeiten (vgl. Brücker et al., 2018). Die Ergebnisse zeigen, dass unabhängig von den heterogenen Kompetenzen die Geflohenen sich durch eine ausgeprägte Erwerbsorientierung auszeichnen, obwohl nur ein Viertel der befragten Geflohenen mit einem Ausbildungs- oder Studienabschluss aus der Heimat zum Zeitpunkt der Untersuchung eine Abschlussanerkennung beantragt hat. Die quantitativen Ergebnisse der zweiten Befragungswelle der IAB-BAMF-SOEP-Befragung verdeutlichen zudem, dass die Erwerbstätigkeitsquote zwar mit der Aufenthaltsdauer steigt (Brücker et al., 2019, S. 9), die Beschäftigung aber tendenziell im Niedriglohnsektor anzusiedeln ist, weil durchschnittliche Bruttomonatsgehälter von vollzeitbeschäftigten Befragten 1.600 EUR nicht übersteigen. Neben denjenigen, die ihrer Qualifikation entsprechend beschäftigt sind (44 Prozent), und jenen, die Berufe mit einem höheren Anforderungsniveau als ihre anerkannte Qualifikation ausüben (25 Prozent), stellen die Autoren auch fest, dass ein nicht unbeträchtlicher Anteil der Geflohenen in Deutschland unterhalb ihrer formellen Qualifikation tätig ist (31 Prozent). Dabei handelt es sich vor allem um Akademikerinnen und Akademiker, die trotz Expertenkenntnisse Helfertätigkeiten erbringen (Brücker et al., 2019, S. 11). Auch regionale Untersuchungen belegen, dass der Einstieg von Geflohenen in den deutschen Arbeitsmarkt sich vorwiegend über die Unterbeschäftigung, wie befristete, Teilzeit- oder geringfügige Beschäftigung, sowie über Qualifizierungsmaßnahmen, wie Praktika und Ausbildung, gestaltet (Hartmann et al., 2018, S. 7). Eine der wenigen qualitativen Studien zur Perspektive von Geflohenen legen Held, Hackl und Bröse (2018) vor. Mit Hilfe von ethnografischen Beobachtungen und qualitativen Interviews untersucht das Forscherteam junge Geflohene in Bezug auf ihre Handlungsorientierungen und stellen dabei fest, dass der arbeitsrechtliche Status und der Spracherwerb die hauptsächlichen Hürden eines Berufseinstiegs darstellen (Held et al., 2018). 
Erkenntnisse darüber, wie bei einem erfolgten Berufseinstieg in Deutschland die Geflohenen ihre betriebliche Integration erleben, welche Maßnahmen Betriebe dafür ergreifen, liegen bislang kaum vor. Nachfolgend wollen wir die Ergebnisse einer explorativen Studie hierzu vorstellen.

\section{Betriebliche Integration: Theoretische Überlegungen}

Das Konzept der beruflichen Integration kann zwischen der organisatorischen Einarbeitung, dem sogenannten Onboarding, und der organisationalen Sozialisation verortet werden. Während die Einarbeitung neuer Beschäftigten im Kontext des Personalmanagements stärker auf ein funktionales Integrieren in das aufgabenbezogene und soziale Umfeld einer Organisation abstellt (vgl. Eisele \& Doyé, 2010, S. 161), wird bei der organisationalen Sozialisation der kulturelle und wertebezogene Aspekt hervorgehoben. So umfasst Landes zufolge organisationale Sozialisation einen „Prozess der Eingliederung von Personen in eine Organisation und damit deren Wandel von einem organisationalen Außenseiter zu einem effektiven Mitglied“ (Landes, 2018, S. 129). Die formalen-funktionalen und die werteorientierten Aspekte der Einarbeitung vereint Brenner (2014) in ihrem Modell, indem sie betriebliche Integration auf drei Ebenen betrachtet: fachliche, soziale und werteorientierte Integration. Dabei stellt die fachliche Integration eine Einarbeitung in das funktionale Arbeitsgebiet dar und beruht auf dem Erwerb von aufgabenbezogenen Kenntnissen und Fähigkeiten. Die soziale Integration wiederum beinhaltet das Aufbauen von sozialen Kontakten in einem Betrieb, wie die zu Vorgesetzten, Arbeitskolleginnen und -kollegen sowie Kundschaft. Als Indiz einer erfolgreichen sozialen Integration wird das Herausbilden eines „Wir-Gefühls“ im Betrieb angesehen (vgl. Brenner, 2014, S. 7). Bei der werteorientierten Integration wiederum handelt es sich um einen Prozess, bei dem Ziele, Werte, Führungsgrundsätze und das Selbstverständnis eines Unternehmens den neuen Beschäftigten vermittelt werden. Als erfolgreich gilt die betriebliche Integration, wenn alle drei Integrationsbestandteile erfüllt sind (vgl. Brenner, 2014, S. 7). Dementsprechend gehört es zu einer systematischen betrieblichen Integration, dass sowohl fachliche, soziale als auch werteorientierte Aspekte adressiert werden.

Üblicherweise stellt die Debatte um betriebliche Integration auf einheimische Beschäftigte als Zielgruppe ab. Mitarbeiterinnen und Mitarbeiter aus dem Ausland werden hierbei selten adressiert. Jedoch empfiehlt Becker
(2015), im Fall einer betrieblichen Integration von Beschäftigten mit Migrationshintergrund zielgerechte Maßnahmen einzusetzen, wie die Möglichkeit des Erwerbs oder die Verbesserung der Deutschkenntnisse oder Mentoringprogramme sowie Tandems zwischen den zugewanderten und den einheimischen Beschäftigten (vgl. Becker, 2015, S. 395).

\section{Methode}

Es wurde eine qualitative explorative Studie auf Basis von halb-strukturierten Interviews mit Geflohenen, die in Deutschland beschäftigt sind, durchgeführt. Als Grundlage für die Interviews wurde ein Leitfaden entwickelt, der mehrere Unterthemen abbildet: persönliche Daten und den beruflichen Werdegang der Geflohenen; Fragen zur aktuellen Position der Geflohenen und zum Berufseinstieg in Deutschland; die betriebliche Integration und die Diversität im Unternehmen sowie Fragen zur persönlichen Meinung der Geflohenen in Bezug auf die betriebliche Integration. Es wurden Leitfadeninterviews mit sechs Geflohenen durchgeführt. Die folgende Tabelle dient zur Übersicht über die Interviewpersonen.

Für die Auswahl der Interviewpartnerinnen und -partner war entscheidend, dass es sich dabei um Personen handelt, die aus ihrem Heimatland geflohen sind, sich in Deutschland niedergelassen und hier bereits Arbeitserfahrung gesammelt haben. Der Kontakt zu den Interviewpersonen wurde durch Angehörige oder Freunde hergestellt. Die Teilnahme an den Interviews erfolgte freiwillig und ohne Vergütung. Vier der Interviews (IP1-4) wurden in einem Café bzw. einer Bäckerei durchgeführt, zwei weitere in der Wohnung der Befragten. Dies schlug sich mitunter in der Interviewdauer nieder - die ersten vier Interviews fielen deutlich kürzer aus -, was nahelegt, dass öffentliche Räume, wie Cafés, im Fall von geflohenen Studienteilnehmerinnen und -teilnehmern nicht oder nur bedingt geeignet sind.

Vor dem Interview wurden die Interviewpersonen über den Hintergrund der Befragung aufgeklärt und haben der Aufzeichnung des Interviews zugestimmt. Alle Interviewpersonen sind in Nordrhein-Westfalen ansässig und hier beruflich tätig.

Die gewonnene Stichprobe ist durchaus heterogen sowohl in Bezug auf die demografischen Merkmale als auch hinsichtlich der Qualifikationen. Es handelt sich um eine Frau und fünf Männer im Alter zwischen 21 und 41 Jahren. Die meisten der Interviewpersonen haben bereits Arbeitserfahrungen in ihrem Heimatland im Vorfeld der Flucht sammeln können. Diese beziehen sich hauptsächlich auf Erfahrungen im handwerklichen oder niedrig 
Tabelle 1. Übersicht über die Interviewpersonen 1-6

\begin{tabular}{|c|c|c|c|c|c|c|}
\hline & IP 1 & IP 2 & IP 3 & IP 4 & IP 5 & IP 6 \\
\hline Geschlecht & $\mathrm{m}$ & $\mathrm{m}$ & $\mathrm{m}$ & $\mathrm{m}$ & w & $\mathrm{m}$ \\
\hline Alter & 21 & 26 & 40 & 41 & 32 & 33 \\
\hline Herkunftsland & Syrien & Syrien & Iran & Irak & Syrien & Syrien \\
\hline $\begin{array}{l}\text { Aufenthalt in } \\
\text { Deutschland seit }\end{array}$ & 2015 & 2015 & 2015 & 2015 & 2015 & 2015 \\
\hline $\begin{array}{l}\text { Bildungsabschluss im } \\
\text { Heimatland }\end{array}$ & Ausbildung & - & Ausbildung & Ausbildung & Studium & Ausbildung \\
\hline $\begin{array}{l}\text { Beruf } \\
\text { Herkunftsland }\end{array}$ & Friseur & - & $\begin{array}{l}\text { LKW-Fahrer/ La- } \\
\text { gerarbeiter }\end{array}$ & Fliesenleger & Lehrerin & Elektriker \\
\hline $\begin{array}{l}\text { Tätigkeit in Deutsch- } \\
\text { land }\end{array}$ & Servicekraft & Saunameister & $\begin{array}{l}\text { Helfer in einer } \\
\text { Baufirma }\end{array}$ & $\begin{array}{l}\text { Produktionshelfer } \\
\text { in Leiharbeit }\end{array}$ & $\begin{array}{l}\text { Betreuerin in } \\
\text { einer OGS }\end{array}$ & $\begin{array}{l}\text { Auszubildender } \\
\text { in einem Maler- } \\
\text { betrieb }\end{array}$ \\
\hline $\begin{array}{l}\text { Tätig im Unternehmen } \\
\text { seit }\end{array}$ & 2019 & 2017 & 2016 & - & 2019 & 2018 \\
\hline Unternehmensgröße & $\begin{array}{l}\text { Mittelständisches Un- } \\
\text { ternehmen (<500 Mit- } \\
\text { arbeiter/-innen) }\end{array}$ & $\begin{array}{l}\text { Kleines Unter- } \\
\text { nehmen (<50 Mit- } \\
\text { arbeiter/-innen) }\end{array}$ & $\begin{array}{l}\text { Kleines Unter- } \\
\text { nehmen (<50 Mit- } \\
\text { arbeiter/-innen) }\end{array}$ & $\begin{array}{l}\text { Große Unterneh- } \\
\text { men (>500 Mitar- } \\
\text { beiter/-innen) }\end{array}$ & $\begin{array}{l}\text { Kleines Unter- } \\
\text { nehmen (<50 Mit- } \\
\text { arbeiter/-innen) }\end{array}$ & $\begin{array}{l}\text { Kleines Unter- } \\
\text { nehmen (<50 Mit- } \\
\text { arbeiter/-innen) }\end{array}$ \\
\hline Dauer des Interviews & 12:24 Minuten & 11:58 Minuten & 12:22 Minuten & 15:49 Minuten & \multicolumn{2}{|l|}{ 40:04 Minuten } \\
\hline Ort des Interviews & Café & Café & Café & Bäckerei & \multicolumn{2}{|c|}{ Wohnung der IP 5 und 6} \\
\hline $\begin{array}{l}\text { Sprachniveau (per- } \\
\text { sönliche Einschätzung } \\
\text { der Interviewerin) }\end{array}$ & B1 & $\mathrm{B} 2-\mathrm{C} 1$ & B1 & B1 & \multicolumn{2}{|l|}{$\mathrm{B} 2-\mathrm{C} 1$} \\
\hline
\end{tabular}

qualifizierten Bereich, wie Friseurin und Friseur, Fliesenlegerin und Fliesenleger, Elektrikerin und Elektriker (IP 1, 4, 6 entsprechend) oder Lastkraftwagenfahrerin und Lastkraftwagenfahrer (IP 3). Drei der befragten Geflohenen gaben an, dass sie in ihrem Heimatland ein Studium begonnen haben, jedoch hat nur Interviewperson 5 dieses auch abgeschlossen und ist danach als Geschichtslehrerin in einer Schule tätig gewesen. Die Interviewperson 2 ist hingegen die einzige, die nicht in ihrem Heimatland berufstätig war, da nur ein Studium begonnen, aber nicht abgeschlossen wurde.

In den Interviews kam es zu verschiedenen sprachlich bedingten Schwierigkeiten. Diese konnten meist durch Nachfragen und erneute oder einfachere Erklärungen gelöst werden. Wenn dies nicht half, kamen Online-Übersetzungsprogramme zur Hilfe (Google Übersetzer und Pons), welche die ursprüngliche Frage in die Muttersprache der Interviewperson übersetzt hat.

Die Auswertung der Leitfadeninterviews erfolgte mit Hilfe der qualitativen Inhaltsanalyse (Gläser \& Laudel, 2010). Das Kodierungsschema richtete sich im Wesentlichen nach den Themen des Interviewleitfadens: die Qualifikationen der Geflohenen, die Berufsanerkennung in Deutschland, aktuelle berufliche Position der Geflohenen sowie die betriebliche Integration, die drei Unterkategorien umfassen: fachliche, soziale und werteorientierte Integration.

\section{Ergebnisse}

\section{Berufseinstieg in Deutschland}

Alle bis auf eine Interviewperson haben bereits Berufserfahrung in ihren Heimatländern, jedoch kann keine der befragten Personen einen formal in Deutschland anerkannten Abschluss vorweisen. Das liegt daran, dass in den Heimatländern - im Unterschied zu Deutschland - eine Ausbildung gänzlich anders abläuft. So ist in Syrien, im Irak und Iran, den Heimatländern der Studienteilnehmerinnen und -teilnehmer, einerseits schulische Berufsausbildung an den Sekundar- oder beruflichen Oberschulen möglich, wiewohl sie teilweise nicht klar von der allgemeinen Schulbildung getrennt ist. Andererseits kann vor allem in Syrien und im Irak eine Ausbildung auch informell direkt im Betrieb absolviert werden, dann aber nur im Rahmen der praktischen Tätigkeit und ohne einen schulischen Unterricht (BQ-Portal, 2020a, b, c). Die berufsrelevanten Tätigkeiten werden dann von anderen Mitarbeiterinnen und Mitarbeitern, Vorgesetzten oder Familienmitgliedern, wie im Fall von Interviewperson 1, gezeigt und dadurch erlernt. Nach einer gewissen Zeit beginnt die lernende Person allein zu arbeiten und kann sich bei Fragen an die oder den Vorgesetzten wenden.

Dementsprechend mussten vier der befragten Interviewpersonen (IP 1, 3, 4 und 6) die Erfahrung machen, 
dass ihre Berufsausbildung in Deutschland nicht anerkannt wird. Um den in ihrer Heimat erlernten Beruf in Deutschland zu ergreifen, müssen sie eine Berufsausbildung hierzulande abschließen. Die anderen zwei Interviewpersonen hatten entweder keinen Berufsabschluss im Heimatland (IP 2) oder bemühten sich um die anteilhafte Anerkennung des heimatlichen Studienabschlusses, um dem Beruf in Deutschland nachgehen zu können (IP 5). Im letzten Fall handelt es sich um den Beruf der Geschichtslehrerin: Die Interviewperson muss in Deutschland Teile des Studiums wiederholen, um weiterhin als Geschichtslehrerin tätig sein zu dürfen.

Durch die fehlende Anerkennung der Bildungsabschlüsse aus den Heimatländern verlief der Berufseinstieg für die Geflohenen sehr unterschiedlich. Die meisten Interviewten eint die Erfahrung der minderwertigen Beschäftigungsverhältnisse. Die eine Hälfte der Interviewten haben den Berufseinstieg in Deutschland über Praktika erlangt, und zwar im eigenen Beruf als Friseur (IP 1) oder über Praktika in verschiedenen Bereichen (IP 5). In beiden Fällen führten Praktika zu einer aktuell ausgeübten geringfügigen Beschäftigung (auf 450-EUR-Basis) als Servicekraft in einem Café (IP 1) oder als Schulbetreuerin (IP 5). In einem Fall diente das Praktikum als Einstiegsqualifizierung für die Überleitung in die Ausbildung als Malermeister unter Anrechnung der Zeit des Praktikums auf die Ausbildungsdauer (IP 6). Die andere Hälfte der Interviewten hat ihren beruflichen Einstieg in den deutschen Arbeitsmarkt zwar ohne Praktika gefunden, ist aber von Beginn an in niedrigqualifizierten und geringfügigen Tätigkeiten eingesetzt, die ebenfalls mit einem erheblichen Prekaritätspotenzial einhergehen. Diese Arbeitsstellen wurden entweder aufgrund der Eigeninitiative gefunden, wie im Fall der Interviewperson 3, die bei einer Baufirma vorstellig wurde und ein Arbeitsangebot für eine Helfertätigkeit erhielt, oder über eine Arbeitsvermittlung durch das Jobcenter - hier handelte es sich um eine geringfügige Beschäftigung als Saunameister (IP 2). In einem weiteren Fall geht es um eine Beschäftigung als Produktionshelfer bei einer Leiharbeitsagentur (IP 4).

\section{Wahrgenommene Arbeitsbedingungen von Geflohenen}

Der Einstieg in den deutschen Arbeitsmarkt erleben alle Befragten als eine Herausforderung. Die Sprache stellt sich als eine besonders schwere Hürde heraus. Bereits die Arbeitssuche und der Bewerbungsprozess werden aufgrund der sprachlichen Barriere als besonders beschwerlich erlebt. Aber auch bei einem geglückten beruflichen Einstieg bleibt die Sprachbarriere bestehen und führt mitunter dazu, dass sich die Geflohenen aufgrund der Sprache an ihrem Arbeitsplatz fremd fühlen (IP 5). Einige der Befragten thematisieren, dass die schriftlichen Formalia, wie Entgeltabrechnungen oder Anträge, eine Schwierigkeit darstellen, da diese schwer zu verstehen sind. Insbesondere dann, wenn die Entgeltabrechnung nicht korrekt ist oder sich das Entgelt mit den wechselnden Einsatzorten ändert, wie im Fall der Interviewperson 4, führen sprachliche Erschwernisse mitunter zum Konfliktpotenzial.

Einen der brisanten Beschäftigungsaspekte für geflohene Personen stellt die erfahrene (Un)gerechtigkeit am Arbeitsplatz dar, insbesondere in Bezug auf den Lohn und die Arbeitstätigkeiten. Vier der sechs interviewten Personen gaben an, mit der Entlohnung für ihren Job zufrieden zu sein. Eine der Interviewpersonen (IP 4) vermerkte zudem, dass sie regelmäßige, von der Betriebszugehörigkeit abhängige Gehaltserhöhungen erwarten kann. Da es jedoch bei den Lohnabrechnungen immer wieder Probleme und Fehler seitens der Leiharbeitsagentur gab, ist die Interviewperson mit dem Gehalt dennoch unzufrieden. Ähnlich wie die Interviewperson 6, aus deren Sicht das Entgelt für das Praktikum sehr gering ausfällt. Gleichwohl sagen fünf der sechs Befragten aus, dass sie eine Gleichbehandlung gegenüber den deutschen Kolleginnen und Kollegen in der vergleichbaren Position erfahren. Die Aufgaben wie auch das Entgelt für die Tätigkeit sind laut den Interviewpersonen gleich und weisen keine Unterschiede zu den einheimischen Kolleginnen oder Kollegen auf. Das gilt auch für die als niedrig empfundene Praktikumsentlohnung im Fall von Interviewperson 6, weil in dem Ausbildungsbetrieb alle Praktikantinnen und Praktikanten gleich wenig verdienen. Eine Ausnahme in Bezug auf die Gleichbehandlungserfahrung stellt die Interviewperson 3 dar, die von den Unterschieden zu den deutschen Kolleginnen und Kollegen trotz der gleichen Position berichtet. Aus seiner Sicht haben die deutschen Kolleginnen und Kollegen immer die gleichen Aufgaben und werden besser behandelt, während er immer unterschiedliche, vor allem bei den deutschen Kolleginnen und Kollegen unbeliebte Aufgaben übernehmen müsse.

\section{Erfahrungen der betrieblichen Integration}

Ausgehend von dem im vorherigen Kapitel vorgestellten theoretischen Modell werden wir im Nachfolgenden die Interviewergebnisse in Bezug auf die betriebliche Integration anhand ihrer drei Komponenten analysieren.

\section{Fachliche Integration}

Die interviewten Personen sind tendenziell in niedrigqualifizierten Tätigkeiten beschäftigt, sagen aber aus, dass ihre 
Arbeit ihren Qualifikationen und Fähigkeiten entspricht und ihnen keine fachlichen Probleme bereitet. Dabei können nur zwei der sechs Befragten (IP 1 und 5) auf bisherige berufsrelevante Erfahrungen zurückgreifen, die sie bereits in anderen Unternehmen in Deutschland als Servicekraft oder in ihrem Heimatland als Lehrerin gesammelt haben. Dementsprechend ist in den meisten Fällen keine aufwendige fachliche Einarbeitung vonnöten.

Zugleich sagen die meisten der Interviewpersonen aus, dass ihnen in den Unternehmen, bei denen sie tätig sind, weder spezielle Fördermaßnahmen zur betrieblichen Integration noch Schulungs- oder Fortbildungsangebote unterbreitet wurden. Zwei der befragten Personen (IP 5 und 6) wurden von ihrem Unternehmen bezahlte Schulungen als Vertiefung bestehender Kompetenzen in Aussicht gestellt, und zwar im Rahmen einer möglichen Ausbildung. Lediglich in einem Fall (IP 2) konnte der Befragte noch während der Probearbeit an einer Schulung zusammen mit der restlichen Unternehmensbelegschaft teilnehmen, die die befragte Person als äußerst nützlich empfand.

Gleichzeitig weisen alle befragten Geflohenen darauf hin, dass ihre Kolleginnen und Kollegen sie in ihre Aufgabe eingearbeitet hätten und sie bei Fragen weiterhin zur Verfügung stünden, unabhängig davon, wie herausfordernd fehlende Sprachkenntnisse sein mögen. Durch Anleitungen und Hilfestellungen seitens der Kolleginnen und Kollegen konnten diese ebenfalls weitestgehend überwunden werden. So berichten alle interviewten Personen, dass sie sich bei Fragen zu den betrieblichen Aufgaben, Strukturen und Abläufen an ihre Kolleginnen oder Kollegen wenden können. Auch Lösungen für die am Arbeitsplatz auftretenden Probleme werden zusammen mit den Kolleginnen und Kollegen gesucht. Bei zwei Interviewpersonen (IP 2 und 3) spielen zusätzlich die Vorgesetzten eine wichtige Rolle bei der Einarbeitung in die betrieblichen Aufgaben und Strukturen und fungieren weiterhin als Ansprechpersonen bei Fragen rund um die Arbeit.

\section{Soziale Integration}

In dieser Studie machen wir die soziale Integration anhand der folgenden Faktoren fest: die Zusammenarbeit mit den Kolleginnen und Kollegen, die Zufriedenheit in Bezug auf die Zusammenarbeit, die Akzeptanz und die Anerkennung der Geflohenen durch die betrieblichen Kolleginnen und Kollegen.

Von allen Interviewpersonen wird die Zusammenarbeit mit den Kolleginnen und Kollegen insgesamt als gut bezeichnet. Alle sind mit der kollegialen Zusammenarbeit zufrieden. Dies scheint nicht einmal der Umstand zu trüben, dass den Geflohenen mitunter Aufgaben zufallen, die von den deutschen Kolleginnen und Kollegen gemie- den werden, wie IP 3 erwähnt. Offenbar fallen solche Erfahrungen subjektiv kaum ins Gewicht, weil dessen ungeachtet alle Befragten aussagen, dass sie sich durch ihre Kolleginnen und Kollegen als Mitglied der Belegschaft akzeptiert fühlen und ihre Leistung als anerkannt empfinden. Sie machen es daran fest, dass sie Lob für ihre Leistung seitens der Kolleginnen und Kollegen, Vorgesetzten und mitunter von Kundinnen und Kunden (wie z.B. im Fall von IP 2 von Gästen der Sauna) erfahren. Nicht zuletzt werden die persönlichen Freundschaften am Arbeitsplatz als ein Normalzustand hervorgehoben:

„Wir sind alle befreundet miteinander und ja, soweit ist alles gut." (IP 2).

\section{Werteorientierte Integration}

Da Maßnahmen des Diversitätsmanagements in einem Unternehmen mitunter die kulturellen und interkulturellen betrieblichen Werte zum Ausdruck bringen, wurde in den Interviews gefragt, inwiefern ein Diversitätsmanagement im aktuellen Unternehmen vorhanden ist, welche Tätigkeiten dabei unternommen werden und inwiefern es als Anlaufstelle für die Interviewpersonen fungiert. Obwohl die interviewten Personen in Unternehmen unterschiedlicher Größe tätig sind, die von kleinen familiengeführten und mittelständischen Unternehmen bis hin zu großen Unternehmen reichen, geben sie alle an, keine Maßnahmen im Bereich Diversitätsmanagement von ihren Arbeitgeberinnen und Arbeitgebern erhalten zu haben. Entsprechend sehen die Befragten ihre Arbeitgeberinnen und Arbeitgeber auch nicht als relevante Ansprechpartnerinnen und -partner in Bezug auf kulturelle Fragen an. Sollte in den großen Unternehmen ein Diversitätsmanagement vorhanden sein, so wurde dieses offenbar nicht an die Arbeitnehmerinnen und Arbeitnehmer kommuniziert.

Neben den (inter)kulturellen Aspekten ist es bei der werteorientierten Integration insbesondere von Bedeutung, inwiefern Betriebe den neuen Beschäftigten ihre Werte vermitteln und mit welchem Erfolg. Hierfür wollen wir uns insbesondere anschauen, inwiefern sich die Geflohenen mit ihren Unternehmen und Aufgaben identifizieren und eine langfristige Perspektive anstreben. Abgefragt wurde dieses anhand der nachfolgenden Fragen: Gibt es Werte, die in dem Unternehmen vermittelt werden? Wurden diese deutlich gemacht? Können Sie sich mit dem Unternehmen und Ihren Aufgaben identifizieren?

Die Hälfte der interviewten Personen (IP 1, 3, und 4) gaben an, dass sie die besonderen Werte der Unternehmen, in denen sie tätig sind, nicht kennen. Die andere Hälfte (IP 2, 5 und 6) sagt aus, dass die Werte, die ihr Unternehmen vertreten, durchaus kommuniziert werden. $\mathrm{Zu}$ solchen wertebezogenen Grundätzen in Unternehmen gehören z.B. die große Relevanz der Entspannung von 
Kundinnen und Kunden, die durch Peelings und Aufgüsse erreicht werden (IP 2), oder aber die Gleichberechtigung aller Arbeitnehmerinnen und Arbeitnehmer sowie die Gleichberechtigung der Kinder (IP 5), oder eine Gleichbehandlung, einhergehend mit einer familiären Beziehung zwischen den Arbeitnehmerinnen und Arbeitnehmern des Unternehmens (IP 6).

Damit korrespondierend sagen zwei der befragten Personen explizit aus, eine ausgeprägte Identifikation mit den Aufgaben und dem Unternehmen zu haben (IP 1 und 2). Trotz der geringfügigen Beschäftigung sind beide Personen gewillt, ihrer Tätigkeit im aktuellen Betrieb auch in der Zukunft nachzugehen, entweder als Hauptberuf oder nebenberuflich neben dem Studium. Ausschlaggebend für die Identifikation ist hierbei vor allem eine zufriedenstellende Zusammenarbeit mit den Kolleginnen und Kollegen sowie die Möglichkeit, die deutsche Sprache während der Arbeit zu erlernen. Die restlichen drei Interviewpersonen haben keine Aussage dazu getroffen (IP 3, 5 und 6). Gleichwohl merken sie an, dass sie eine langfristige Beschäftigung sowie potenzielle Weiterentwicklung oder Beförderung in den aktuellen Betrieben anstreben, wie z. B. eine Tätigkeit als Lehrerin oder eine Fortbildung zum Meister im Anschluss an die Ausbildung.

Eine Interviewperson gibt hingegen an, dass sie sich nicht mit ihrem Unternehmen und der Aufgabe identifizieren kann (IP 4). In diesem Fall ist dies im Wesentlichen auf die Leiharbeitsstruktur zurückzuführen, bei der die stets wechselnden Aufgaben in verschiedenen Unternehmen eine Identifikation erschweren. Auch die bisherigen Probleme mit den fehlerhaften Entgeltabrechnungen seitens des Leihunternehmens verhindern die Identifikation mit dem Arbeitgeber. Vor diesem Hintergrund ist es nachvollziehbar, dass im Sinne einer besseren werteorientierten Integration sich die befragte Person eine bessere Kommunikation sowie ein größeres Verständnis für die Situation der Geflohenen seitens des Unternehmens wünscht.

In allen Fällen wird eine enge Verschränkung der drei Integrationskomponenten sichtbar. Insbesondere die soziale Integration scheint ausschlaggebend für den Erfolg oder Misserfolg der betrieblichen Integration zu sein, wie das nachfolgende Zitat verdeutlicht:

"Ich habe Erfahrungen, meine Kollegen sind nett und besonders die Kinder sind so süß und so lieb." (IP 5).

\section{Zusammenfassung und Diskussion der Ergebnisse}

Das Ziel dieser explorativen Studie bestand darin, die Praktiken der betrieblichen Integration in Deutschland aus der Perspektive der Geflohenen zu beleuchten. Obwohl die sechs durchgeführten qualitativen Interviews nur eine erste explorative Erkundung darstellen, legen sie zwei wesentliche Erkenntnisse nahe. Zum einen stellt es sich heraus, dass der Einstieg in den deutschen Arbeitsmarkt für befragte Geflohene meist in Form von atypischen Beschäftigungsverhältnissen oder -konstellationen geschieht, und zwar durch temporäre Praktika oder eine geringfügige Beschäftigung. Dabei spielen die in den Heimatländern erlangten Kenntnisse offenbar, wenn überhaupt, nur eine marginale Rolle, denn unabhängig von der bisherigen Ausbildung, Studienabschlüssen oder Berufserfahrung werden die Geflohenen allesamt für niedrigqualifizierte und eher schlecht honorierte Tätigkeiten, meist im Helferbereich, eingestellt. Einer der Gründe hierfür ist eine zeitaufwendige und umständliche oder eine gänzlich verwehrte Anerkennung bisheriger Abschlüsse aus den Heimatländern, sofern diese vorliegen. Damit erweist sich der Arbeitsmarkt in Deutschland trotz zahlreicher Liberalisierungstendenzen der letzten Jahrzehnte (s. dazu Etzold, 2018) weiterhin als restriktiv und in einem hohen Maße selektiv den in einem nichteuropäischen Ausland erworbenen Kompetenzen gegenüber. Die in der bisherigen Forschung bezüglich der Arbeitsmigration kritisierte Entwertung der ausländischen Kompetenzen (z. B. Kaabel, 2017) scheint im Fall von Geflohenen nach Deutschland weiterhin zu bestehen, zumindest im Fall der hier befragten Personen. Um eine problemlose Arbeitsmarktintegration zu vollziehen, wird den Geflohenen stattdessen nahegelegt, eine erneute Qualifizierung in Deutschland zu erlangen, wofür sich einige der von uns befragten Geflohenen dann schlussendlich in Form von einer Ausbildung in einem deutschen Betrieb entscheiden. Damit bleiben die arbeitsbezogenen Einkommensmöglichkeiten für Geflohene jahrelang auf einem niedrigen Niveau und eine langfristige Aussicht auf ein steigendes Einkommen besteht nur für diejenigen, die sich die Mühen der Anerkennung, der Überbrückung von Kompetenzen oder einer erneuten Ausbildung auf sich nehmen.

Die zweite empirische Erkenntnis der vorliegenden Studie weist auf die alltägliche Praxis der betrieblichen Integration von Geflohenen hin. Aus Sicht der Teilnehmerinnen und -teilnehmer dieser explorativen Studie sind es nicht die Diversitäts- oder Gleichstellungsbeauftragten, die ihnen bei der Einarbeitung und Eingewöhnung zur Seite stehen. Das sind auch nicht die Referentinnen oder Referenten der Personalabteilungen. Manchmal sind es die Vorgesetzten, die für die Geflohenen wichtige Ansprechpartnerinnen und Ansprechpartner zu arbeitsbezogenen Fragen sind. Am ehesten und am wichtigsten sind für die Geflohenen jedoch ihre Kolleginnen und Kollegen am Arbeitsplatz, die sie in fachlicher, sprachlicher und 
sozialer Hinsicht unterstützen, beraten, unterrichten und bei Problemen helfen. Die als explorativ zu wertenden Erkenntnisse dieser Arbeit legen somit nahe, dass die eigentliche Integrationsarbeit in Betrieben die Fachkolleginnen und -kollegen erbringen, die Seite an Seite mit den Geflohenen die Arbeitsaufgaben bewältigen müssen und ungeachtet der sprachlichen, kulturellen und anderweitigen Hürden Probleme zu lösen versuchen. Trotz ihres explorativen Charakters verdeutlichen die Interviewergebnisse, von welch großer Bedeutung diese kollegiale Unterstützung für die Geflohenen selbst ist: Sie erlernen dabei nicht nur fachliche Kompetenzen, sondern die Unternehmenspraktiken, die arbeitsbezogenen Gepflogenheiten und nicht zuletzt auch die deutsche Sprache. Man kann somit annehmen, dass zumindest in einigen der Betriebe, die die Geflohenen beschäftigen, alltäglich eine informelle multimodale Personalentwicklung durch einheimische Beschäftigte statt, die auf diese Weise einen essentiellen Beitrag zur arbeitsbezogenen wie gesellschaftlichen Integration von Geflohenen leisten. Interessanterweise kommt diese Art der betrieblichen Integration von Geflohenen sehr nah dem in den meisten Herkunftsländern üblichen Format der Ausbildung: informell, basierend auf Beobachtungen und stellvertretendem Lernen am Arbeitsplatz.

Die Ergebnisse dieser explorativen Untersuchung tragen zur bisherigen Forschung insofern bei, dass sie zum einen die bereits diskutierten Herausforderungen der betrieblichen Integration von Geflohenen bestätigen. So werden die sprachlichen und kulturell bedingten Probleme, die in der Forschung oft thematisiert werden (z. B. Flake et al., 2017a; 2017b), auch als eine gravierende Herausforderung für Geflohene selbst herausgestellt, die sowohl die Klärung fachlicher Probleme, wie z.B. fehlerhafte Lohnabrechnungen, wie auch die alltägliche Kommunikation am Arbeitsplatz erschwert. Den bisherigen Arbeiten (z. B. Flake et al., 2017b) ähnlich, legt auch unsere Studie die Verbreitung und die Problematik der niedrigschwelligen Einstiegsoptionen in den Arbeitsmarkt in Form von Praktika oder geringfügiger Beschäftigung für Geflohene nahe. Zum anderen weisen die explorativen Ergebnisse aber auch auf Themen hin, die über die bisherigen Diskussionen in der Forschung hinausgehen. Entgegen der bisherigen Forschungskonzentration auf die Unterstützungsangebote seitens des Staates und der Arbeitgeberinen und Arbeitgeber (z.B. Müller \& Schmidt, 2016) deuten die Ergebnisse dieser Studie an, dass für die Geflohenen selbst die informelle Unterstützung und Integration durch die Arbeitskolleginnen und -kollegen von einer weitaus gravierenderen Bedeutung sind. Dass die "einfachen" Beschäftigten eine wichtige Komponente der betrieblichen Integration darstellen können, wurde sowohl in der bisherigen Forschung wie in der Praxis ver- nachlässigt. Dieser Aspekt bedarf in den zukünftigen Studien sowie Integrationsprojekten einer besonderen Beachtung. Daraus ist auch abzuleiten, dass die Onboarding-Prozesse im Fall von geflohenen Personen einige Besonderheiten beinhalten, die bislang ungenügend berücksichtigt wurden und denen durch differenzierte Onboarding-Modelle Rechnung getragen werden sollte. So sollte das Onboarding nicht nur das Arbeitskollegium als eine wichtige Integrationssäule stärker berücksichtigen, sondern auch viel enger in Verbindung mit den Qualifizierungsangeboten im sprachlichen, fachlichen wie kulturellen (werteorientierten) Bereich gedacht werden.

Limitationen dieser Studie sollten jedoch auch nicht außer Acht gelassen werden. Dazu gehört vor allem die niedrige Anzahl der durchgeführten Interviews. Damit bleibt die Untersuchung rein explorativ und als Ausgangspunkt für weitere, möglicherweise quantitative Untersuchungen. Eine weitere Limitation stellt eine eingeschränkte Aussagefähigkeit der Ergebnisse aufgrund der deutschen Sprache dar. Die Befragten konnten zwar insgesamt ein gutes Gespräch führen, dennoch war es offensichtlich eine Herausforderung für sie und manche Antworten fielen sehr kurz aus oder blieben ganz aus, weil die Fragen entweder nicht verstanden oder als heikel eingeschätzt wurden. Diese sprachlich bedingte Ungewissheit bleibt eine grundsätzliche Herausforderung der qualitativen sprachbezogenen Forschung.

\section{Empfehlungen für die Beschäftigungspraxis von geflohenen Personen}

Die gewonnenen Ergebnisse erlauben es, einige Gestaltungsempfehlungen für Wirtschaftsunternehmen in Bezug auf die betriebliche Integration von Geflohenen abzuleiten. Es wird deutlich, dass das Angebot zur Förderung der betrieblichen Integration in Unternehmen einer Erweiterung bedarf. Im Sinne einer fachlichen Integration sind vor allem betriebsspezifische Sprachkurse, welche das betriebsnotwendige Fachvokabular vermitteln können, von großer Bedeutung, im Unterschied zu den allgemeinen Sprachkursen. Anzudenken ist zudem auch ein Angebot an Schulungen und Weiterbildungen zur Stärkung der fachlichen Kompetenzen von Geflohenen. $\mathrm{Zu}$ beachten wäre hierbei allerdings, nicht nur gesonderte Schulungen für Geflohene anzubieten, sondern auch Schulungen, an denen Geflohene zusammen mit anderen Kolleginnen und Kollegen teilnehmen und das teambezogene Lernen praktizieren können. 
Obwohl oben kritisch thematisiert wurde, dass der berufliche Einstieg für geflohene Personen oft über Praktikum oder eine Unterbeschäftigung erfolgt, ist zugleich anzumerken, dass diese niedrigschwelligen Einstiegsoptionen auch ein Sprungbrett in den ersten Arbeitsmarkt darstellen können (nicht müssen!). Gerade vor diesem Hintergrund liegt eine große Aufgabe für Unternehmen und andere Arbeitsmarktakteurinnen und -akteure weiterhin darin, Geflohene für das spezifische deutsche Ausbildungsmodell zu sensibilisieren und dieses als eine potenzielle und mittelfristige Ermöglichung einer soliden Beschäftigungs- und Einkommensperspektive zu verdeutlichen.

Neben der sprachlichen und fachlichen Förderung ist auch eine gezielte Förderung des Betriebsklimas zugunsten einer sozialen Integration sinnvoll. Dies kann durch gemeinschaftliche Aktivitäten oder Veranstaltungen für die Beschäftigten geschehen. Auch die Vermittlung der betrieblichen Werte an geflohene Mitarbeiterinnen und Mitarbeiter sollten Unternehmen als eine ihrer Aufgaben betrachten, damit die Geflohenen eine Gelegenheit haben, sich mit den betrieblichen Werten, aber auch gesellschaftlichen Grundsätzen sowie den Besonderheiten des Wirtschaftslebens in Deutschland auseinanderzusetzen. Die Sensibilisierung von Mitarbeiterinnen und Mitarbeitern für die Vielfalt der betrieblichen Belegschaft stellt eine genuine Aufgabe des Diversitätsmanagements in Unternehmen dar. Die geflohenen Beschäftigten stellen daher einen überaus konkreten und akuten Anlass für eine Neu- oder Umgestaltung der Maßnahmen zum Diversitätsmanagements dar, um sich der Diversität im betrieblichen Anlass zu vergegenwärtigen, aber auch eine strukturelle Benachteiligung, z. B. in Form von prekäreren Beschäftigungsmodi als einheimische Beschäftigte, kritisch zu hinterfragen.

Eine weitere Empfehlung bezieht sich auf die Kolleginnen und Kollegen, die die betriebliche Integration von Geflohenen am Arbeitsplatz vollbringen. Unternehmen sollten darüber nachdenken, spezielle Unterstützungsangebote für diese Zielgruppe zu unterbreiten, wie z.B. interkulturelle Schulungen oder Workshops zu Konfliktund Problemlösungen. Obwohl die kollegiale Einarbeitung meist auf informellem Wege erfolgt, stellt diese Integrationsarbeit für die betroffenen Beschäftigten einen erheblichen Mehraufwand dar: Es handelt sich nicht nur um eine reine fachliche Einarbeitung von neuen Beschäftigten, sondern auch um ein sprachliches Anlernen sowie eine soziale Betreuung der Geflohenen. Damit diese Mehrarbeit nicht zu negativen Auswirkungen, wie innere Kündigung (Raidt, 1989) oder Gratifikationskrisen von Beschäftigten (Siegrist, 2015), gestiegenen Krankenständen und Fluktuation führt, sollten Unternehmen prüfen, auf welche Art die einheimischen Beschäftigten sinnvol- lerweise honoriert werden könnten. Die Palette der Möglichkeiten, die Mehrarbeit anzuerkennen, ist durchaus breit und reicht von leistungsbezogenen Gehaltsbestandteilen bis hin zu betrieblichen Sozialleistungen. Um eine Überforderung der Mitarbeiterinnen und Mitarbeiter, die informelle Integrationsarbeit vollbringen, zu vermeiden, wären zudem externe Unterstützungsangebote im Bereich kulturelle und betriebliche Integration zu berücksichtigen.

Vor dem Hintergrund der genannten Empfehlungen wird deutlich, dass für die Personalabteilungen in Unternehmen eine neue Bedeutung in Zusammenhang mit der Beschäftigung von Geflohenen zukommen kann. Die Personalressorts in Unternehmen sollten Geflohene als eine spezielle Zielgruppe in ihren Belegschaften betrachten, die einer gesonderten Berücksichtigung bedürfen. Wichtig ist, dass die Personalabteilungen die betriebliche Integration der Geflohenen als ihre Aufgabe ansehen und somit als Ansprechpartnerinnen und Ansprechpartner sowohl für die Geflohenen wie für ihre Führungskräfte und Arbeitskolleginnen und -kollegen agieren. Bedürfnisorientierte betriebliche Maßnahmen der Personalentwicklung in Form von Trainings und Schulungen für einzelne Gruppen wie für komplette Belegschaften können in diesem Kontext ein fester Bestandteil der Personalarbeit werden.

\section{Literatur}

Battisti, M., Felbermayr, G. \& Poutvaara, P. (2015). Arbeitsmarktchancen von Flüchtlingen in Deutschland: Ergebnisse einer Unternehmensbefragung. München: ifo Institut - Leibniz-Institut für Wirtschaftsforschung an der Universität München.

Becker, M. (2015). Systematisches Diversity Management: Konzepte und Instrumente für die Personal- und Führungspolitik. Stuttgart: Schäffer-Poeschel.

Blunck, B. A. (2018). Arbeitsrechtliche Aspekte der beruflichen Integration von Flüchtlingen. In A. Frintrup (Hrsg.), Berufliche Integration von Flüchtlingen und Migranten. Psychologische Kompetenzanalyse, rechtliche Rahmenbedingungen, Prozessgestaltung \& Praxisbeispiele (S. $11-29)$. Berlin: Springer.

BQ-Portal (2020a). Länderprofil Syrien. Verfügbar unter: https:// www.bq-portal.de/db/L\%C3\%A4nder-und-Berufsprofile/syrien

BQ-Portal (2020b). Länderprofil Irak. Verfügbar unter: https:// www.bq-portal.de/db/L\%C3\%A4nder-und-Berufsprofile/irak

BQ-Portal (2020c). Länderprofil Iran. Verfügbar unter: https:// www.bq-portal.de/db/L\%C3\%A4nder-und-Berufsprofile/iran

Brenner, D. (2014). Onboarding, Als Führungskraft neue Mitarbeiter erfolgreich einarbeiten und integrieren. Wiesbaden: Springer Gabler.

Brücker, H., Rother, N., Schupp, J., Babka von Gostomski, C., Böhm, A., Fendel, T., et al. (2016). Flucht, Ankunft in Deutschland und erste Schritte der Integration. DIW Wochenbericht, 46, $1103-1119$.

Brücker, H., Rother, N. \& Schupp, J. (2018). IAB-BAMF-SOEP-Befragung von Geflüchteten 2016: Studiendesign, Feldergebnisse 
sowie Analysen zu schulischer wie beruflicher Qualifikation, Sprachkenntnissen sowie kognitiven Potenzialen (Forschungsbericht Nr. 30). Nürnberg: Bundesamt für Migration und Flüchtlinge.

Brücker, H., Croisier, J., Kosyakova, Y., Kröger, H., Pietrantuono, G., Rother, N. et al. (2019). Zweite Welle der IAB-BAMF-SOEP-Befragung: Geflüchtete machen Fortschritte bei Sprache und Beschäftigung (IAB-Kurzbericht, 3/2019). Nürnberg: IAB, Institut für Arbeitsmarkt- und Berufsforschung

Büschel, U., Hense, C., Daumann, V., Dony, E., Kubis, A., Rebien, M. et al. (2018). Betriebe und Geflüchtete: 36 Interviews in Betrieben im vierten Quartal 2016 (Forschungsbericht Nr. 2.). Nürnberg: IAB, Institut für Arbeitsmarkt- und Berufsforschung

Bundesagentur für Arbeit (2020, März). Berichte: Arbeitsmarktkompakt - Fluchtmigration. Nürnberg: Bundesagentur für Arbeit.

Eilers, S., Möckel, K., Rump, J. \& Schabel, F. (2017). Integration von Flüchtlingen in den deutschen Arbeitsmarkt. Ludwigshafen am Rhein \& Mannheim: Institut für Beschäftigung und Employability IBE und Hays AG.

Eisele, D. \& Doyé, T. (2010). Praxisorientierte Personalwirtschaftslehre. Wertschöpfungskette Personal (7., Aufl.). Stuttgart: Kohlhammer.

Etzold, B. (2018). Arbeit trotz Asyl? Erlebte Chancen und Hürden von Geflüchteten beim Zugang zu Arbeit. In: M. Becker, V. Kronenberg \& H. Pompe (Hrsg.), Fluchtpunkt Integration, Panorama eines Problemfeldes (S. 319 -353). Wiesbaden: Springer VS.

Flake, R., Jambo, S., Pierenkemper, S., Placke, B. \& Werner, D. (2017a). Engagement von Unternehmen bei der Integration von Flüchtlingen: Erfahrungen, Hemmnisse und Anreize. Köln: Institut der deutschen Wirtschaft (IW) \& Kompetenzzentrum Fachkräftesicherung (KOFA).

Flake, R., Jambo, S., Pierenkemper, S., Risius, P. \& Werner, D. (2017b). Beschäftigung und Qualifizierung von Flüchtlingen in Unternehmen: Die Bedeutung von Unterstützungsangeboten bei der Integration. IW-Trends-Vierteljahresschrift zur empirischen Wirtschaftsforschung, 44, 3-20.

Gläser, J. \& Laudel, G. (2010). Experteninterviews und qualitative Inhaltsanalyse als Instrumente rekonstruierender Untersuchungen (4., Aufl.). Wiesbaden: VS Verlag für Sozialwissenschaften.

Gürtzgen, N., Kubis, A. \& Rebien, M. (2017). Geflüchtete kommen mehr und mehr am Arbeitsmarkt an (IAB-Kurzbericht, 14/2017). Nürnberg: Institut für Arbeitsmarkt- und Berufsforschung.

Hartmann, C., Leicht, R. \& Sajons, C. (2018). Potenziale und Hemmnisse der Integration von Geflüchteten in den badenwürttembergischen Arbeitsmarkt (Der Gesellschaftsreport Baden-Württemberg, 4/2018). Stuttgart: Ministerium für Soziales und Integration Baden-Württemberg.
Held, J., Hackl, R. \& Bröse, J. (2018). Berufseinstieg von Geflüchteten. Chancen und Probleme des Übergangs in die Arbeitswelt (Working Paper, 070) Düsseldorf: Hans-Böckler-Stiftung.

Kaabel, A. (2017). Losing human(itarian) capital: An analysis of barriers to and prospects of refugees' labour market integration in Germany. Refugee Review, 3, 46-64.

Landes, A. (2018). Integration hochqualifizierter Migranten durch Organisationen. Wiesbaden: Springer Gabler.

Lundborg, P. \& Skedinger, P. (2016). Employer attitudes towards refugee immigrants: Findings from a Swedish survey. International Labour Review, 155, 315-335.

Meier, C. \& Mager, C. (2019). Integration von Flüchtlingen in Ausbildung und Arbeitsmarkt: Gemeinsam nach vorne schauen. In: A. B. Karlshaus, I. C. Mochmann (Hrsg.), CSR Interkulturelles Management Gesellschaftliche und unternehmerische Verantwortung international bewältigen (S. 243-251). Wiesbaden: Springer Gabler.

Müller, A. \& Schmidt, W. (2016). Fluchtmigration und Arbeitswelt: Maßnahmen zur Integration von Flüchtlingen in großen Unternehmen. (Studie der Hans-Böckler-Stiftung Nr. 339). Düsseldorf: Hans-Böckler-Stiftung.

OECD (2017). Nach der Flucht: Der Weg in die Arbeit - Arbeitsmarktintegration von Flüchtlingen in Deutschland. Berlin: OECD.

Raidt, F. (1989). Innere Kündigung. In H. Strutz (Hrsg.), Handbuch Personalmarketing (S. 68 -82). Wiesbaden: Gabler.

Siegrist, J. (2015). Arbeitswelt und stressbedingte Erkrankungen. Forschungsevidenz und präventive Maßnahmen. München: Elsevier.

\section{Historie}

Eingegangen:22.06.2020

Revision eingegangen:01.12.2020

Onlineveröffentlichung: 12.02.2021

\section{Autorenschaften}

Stefanie Wilkmann, Hochschule Hamm-Lippstadt

\section{ORCID}

Irma Rybnikova

(iDhttps://orgid.org/0000-0002-1162-755X

\section{Prof. Dr. Irma Rybnikova}

Department Lippstadt 2

Hochschule Hamm-Lippstadt

Marker Allee 76-78

59063 Hamm

Irma.Rybnikova@hshl.de 